\title{
Deserción escolar en la educación superior en México: revisión de literatura
}

\section{School drop-off in top education: literature review}

\section{Abandono escolar no ensino superior no México: revisão da literatura}

Fabiola Lydie Rochin Berumen

Universidad Autónoma de Zacatecas, Unidad Académica de Medicina Veterinaria y Zootecnia,

México

fabiolauaz@outlook.com

https://orcid.org/0000-0002-8676-7768

\section{Resumen}

El objetivo de este trabajo fue realizar una revisión de literatura para conocer algunas definiciones, así como las causas que generan la deserción escolar en el nivel universitario. El enfoque metodológico empleado fue cualitativo, para lo cual se tomaron en cuenta los métodos bibliográfico, histórico-lógico, analítico, sintético y descriptivo; el proceso de revisión literaria permitió determinar y extraer la información más destacada contenida en diferentes fuentes, como la ofrecida en los portales Scielo, Redalyc, Dialnet, así como en los buscadores Google Scholar y Mendely. Algunas de las causas señaladas en el presente documento en torno a la deserción escolar tienen que ver con las fallas latentes en los planes y programas de estudio, las deficiencias en la preparación y actualización del personal docente, las dificultades familiares que debe enfrentar el estudiante o la carencia de un objetivo o proyecto de vida de su parte. En conclusión, se puede afirmar que las instituciones de educación superior deben involucrarse de forma más activa en dicho fenómeno, lo cual se puede lograr actualizando sus programas académicos e incluyendo estrategias didácticas, asesorías y seguimiento a los estudiantes mediante tutorías. Asimismo, se 




Revista Iberoamericana para la Investigación y el Desarrollo Educativo

ISSN $2007-7467$

debe trabajar en el aspecto emocional de los estudiantes, para lo cual se debe lograr establecer empatía para facilitar la adaptación, en especial en el primer año universitario.

Palabras claves: causas, deserción escolar, estudiantes, nivel superior.

\begin{abstract}
The objective of this work was to conduct a literature review to learn about some definitions, as well as the causes of dropout at the university level. The methodological approach used was qualitative, for which bibliographic, historical-logical, analytical, synthetic and descriptive methods were taken into account; the literary review process allowed to determine and extract the most outstanding information contained in different sources, such as that offered on the Scielo, Redalyc, Dialnet portals, as well as in the Google Scholar and Mendely search engines. Some of the causes identified in this document around dropout have to do with latent failures in processing plans and programs, deficiencies in teacher readiness and updating, family difficulties facing the student, or lack of a life goal or project on their part. In conclusion, it can be said that higher education institutions must become more actively involved in this phenomenon, which can be achieved by updating their academic programs and including teaching strategies, counseling and follow-up to students through tutoring. In addition, work must be done on the emotional aspect of students, for which empathy must be established to facilitate adaptation, especially in the first year of university.
\end{abstract}

Keywords: causes, dropout, students, higher level.

\title{
Resumo
}

O objetivo deste trabalho foi realizar uma revisão da literatura para conhecer algumas definições, bem como as causas que geram a evasão escolar no nível universitário. A abordagem metodológica utilizada foi qualitativa, para a qual foram considerados os métodos bibliográfico, histórico-lógico, analítico, sintético e descritivo; O processo de revisão literária permitiu determinar e extrair as informações mais relevantes contidas em diferentes fontes, como a oferecida nos portais Scielo, Redalyc, Dialnet, bem como nos buscadores Google Scholar e Mendely. Algumas das causas apontadas neste documento em torno do abandono escolar dizem respeito a falhas latentes nos planos e programas de estudos, deficiências na preparação e atualização do corpo docente, 


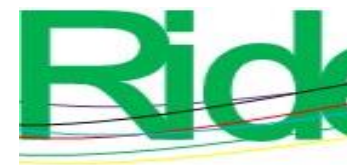

Revista Iberoamericana para la Investigación y el Desarrollo Educativo

ISSN $2007-7467$

dificuldades familiares que o aluno deve enfrentar ou a falta de um objetivo ou projeto de vida de sua parte. Em conclusão, pode-se afirmar que as instituições de ensino superior devem estar mais ativamente envolvidas neste fenômeno, o que pode ser alcançado atualizando seus programas acadêmicos e incluindo estratégias de ensino, orientando e acompanhando os alunos por meio de tutorias. Da mesma forma, é necessário trabalhar o aspecto emocional dos alunos, para o qual deve ser possível estabelecer empatia para facilitar a adaptação, principalmente no primeiro ano da universidade.

Palavras-chave: causas, evasão escolar, alunos, nível superior.

Fecha Recepción: Abril 2020

Fecha Aceptación: Enero 2021

\section{Introducción}

La deserción escolar es una problemática que afecta a estudiantes de todo el mundo, de ahí que sus consecuencias terminen impactando al conjunto de la sociedad. Por eso, en el presente estudio se procura realizar una revisión literaria sobre dicho fenómeno en la educación superior, el cual debe ser enfrentado no solo por los estudiantes, sino también por las distintas instituciones educativas, las cuales deben intentar conocer y atender las dificultades que en muchos casos obligan a los alumnos a abandonar su proceso formativo (Latiesa, 2011). Algunas de las causas señaladas en el presente documento tienen que ver con las fallas latentes en los planes y programas de estudio (Ortiz-Hernández, 2018), las deficiencias en la preparación y actualización del personal docente, las dificultades familiares que debe enfrentar el estudiante o la carencia de un objetivo o proyecto de vida (Vries, León Arenas, Romero Muñoz y Hernández Saldaña, 2011).

\section{Método}

El enfoque metodológico empleado fue cualitativo, para lo cual se tomaron en cuenta los métodos bibliográficos, histórico-lógico, analítico, sintético y descriptivo; el proceso de revisión literaria permitió determinar y extraer la información más destacada contenida en diferentes fuentes, como la ofrecida en los portales Scielo, Redalyc, Dialnet, Academia.edu, así como en los buscadores Google Scholar y Mendely. En eje de la búsqueda de información se llevó a cabo en torno a deserción escolar y causas de la deserción escolar en el nivel superior. 


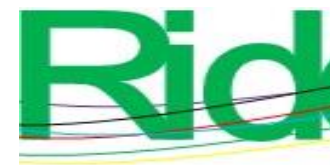

Revista Iberoamericana para la Investigación y el Desarrollo Educativo ISSN $2007-7467$

\section{Resultados}

A partir de la revisión de la literatura, se puede decir que los estudiantes abandonan la escuela debido a factores de diversa índole, como personales, vinculados con la falta de motivación, relaciones interpersonales, embarazos en adolescentes, etc., problemas socioeconómicos asociados con el nivel de ingreso familiar; y pedagógicos, relacionados con las estrategias, actividades, recursos y evaluación académica.

Es importante analizar los factores que influyen en la deserción escolar a nivel universitario. Para ello, se debe atender a todos los estudiantes que puedan presentar algún tipo de inconveniente, en especial a los de nuevo ingreso, pues estos se enfrentan a un ámbito académico que les exige retos y responsabilidades con los que no están familiarizados. En concordancia con esta idea, Silva-Laya (2011) demuestra que el primer año universitario constituye un tramo crítico en la definición de trayectorias estudiantiles exitosas, pues es en este lapso donde se suele tomar la decisión de abandonar o proseguir con los estudios. Los alumnos, por tanto, deben ser acompañados para que enfrenten las dificultades externas (p. ej., relacionadas con los programas académicos), así como las variables personales vinculadas sobre todo con la autoestima. Para eso, los padres de familia también deben involucrarse, pues ellos representan un apoyo invaluable para los hijos, lo cual impactaría de forma positiva en la motivación, conducta y, por ende, rendimiento académico (Espinosa Hoffmann y Claro Stuardo 2010).

Otra figura que se debe reforzar es la del tutor académico, papel que debe ser desempeñado con el fin de llevar un seguimiento y un análisis del proceso de aprendizaje del alumnado para prevenir la deserción escolar. En tal sentido, los centros educativos deben implementar un plan de acción tutorial que incluya asesoramiento psicológico, lo cual de seguro disminuirá los índices de deserción escolar (Muñoz y Martínez, 2017).

\section{Deserción escolar}

Definir este concepto de forma puntual resulta complejo debido a que no existen parámetros teóricos claros que lo delimiten, más allá del indicador con el que se refiere al ausentismo o abandono de un joven de la institución donde se matriculó para cursar el año escolar. Esto trae como consecuencia que tanto para investigadores como para creadores de políticas públicas resulte difícil emprender acciones que permitan disminuir su impacto (Fernández, 2010). 


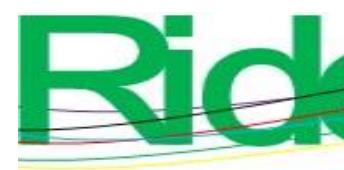

Revista Iberoamericana para la Investigación y el Desarrollo Educativo

ISSN $2007-7467$

Aun así, autores como Spady (1970) parten de la idea general de asociar la deserción escolar universitaria con cualquier persona que se retira de una institución educativa antes de recibir su diploma. Para Gómez (1998), esta ruptura constituye "un fracaso personal temprano cuya marca dura para toda la vida” (p. 54). Para Frankiln y Kochan (2000) este concepto se genera cuando una persona, inscrita en alguna carrea el año anterior, decide no continuar con sus estudios sin haber sido transferida a otra escuela.

Vásquez Velásquez, Castaño Vélez, Gallón Gómez y Gómez Portilla (2003), tomando como base la heterogeneidad del concepto, lo analizan desde el punto de vista temporal y espacial. En el primer caso, lo relacionan con el tiempo que dura un estudiante en un programa académico hasta que se retira, lo cual sirve para identificar tres tipos de deserción: precoz, temprana y tardía. Por otra parte, la vinculación espacial del concepto se produce cuando el estudiante cambia de programa dentro de una misma institución, cuando cambia de institución educativa e incluso cuando sale del sistema educativo, pero con la posibilidad de continuar en el futuro, sea en la misma institución o en otra.

En concordancia con esta idea, el Departamento Nacional de Estadística (DANE) (2016) señala que la deserción se produce cuando el estudiante se desvincula definitiva o temporalmente del sistema educativo formal. Esta idea también es apoyada por Lavaros y Gallegos (2005), aunque estos autores consideran que dicho fenómeno no se podría considerar como tal cuando ha sucedido por los motivos asociados a enfermedades, accidentes u obligaciones legales (p. ej., el servicio militar obligatorio). Aunado a esto, Del Castillo (2012) opina que la deserción escolar también tiene relación con el abandono de los sueños y perspectivas de una vida futura provechosa y responsable.

Por eso, se dice que la deserción escolar es un problema multicausal que involucra no solo a factores intrínsecos del individuo, sino también a variables familiares, económicas, contextuales y educativas (De Witte y Rogge, 2013; Páramo y Correa, 2012; Vázquez, 2012).

\section{Causas de la deserción escolar}

Los modelos y las teorías que han examinado el problema de la deserción escolar se han enfocado en evaluar las características académicas, socioeconómicas, psicológicas y familiares de los estudiantes cuando ingresan a la educación superior (Arango, 2007). Así, autores como Santamaría y Bustos (2013) señalan que las principales causas de la deserción se vinculan con las 




Revista Iberoamericana para la Investigación y el Desarrollo Educativo

ISSN 2007 - 7467

deficiencias en cuanto a la preparación académica previa de los estudiantes, lo cual dificulta el proceso de integración social en el nuevo nivel educativo.

Rinne y Järvinen (2011), por su parte, explican que la confianza académica del individuo influye en la decisión de desertar, mientras que Román (2013) afirma que la mayor parte de los estudiantes que se retiran del sistema educativo lo hace por razones financieras, familiares y bajos rendimientos académicos. Entre las variables sociales y económicas estudiadas se encuentran la desigualdad social y económica (Rosado-Ortiz, 2012), mientras que autores como Torres (2012) señalan la responsabilidad que subyace en los programas académicos y su falta de actualización.

Sobre los factores económicos, Donoso y Schiefelbein (2012) indican que en muchos casos afecta la percepción del estudiante acerca de su capacidad o incapacidad para solventar los costos asociados a los estudios universitarios, lo cual puede ser atendido mediante créditos a largo plazo, tasas relativamente blandas, subsidios parciales o totales, así como becas de matrícula y de alimentación, etc. Por eso, Moreno Bernal (2013) considera que deserción también puede ser promovida, paradójicamente, por la misma institución educativa, la cual, debido a los altos costos de matrículas, impide a la población de bajos recursos continuar con sus estudios.

En este sentido, el INEE (2017) advierte que los problemas relacionados con la deserción escolar son causados por factores intersistémicos e intrasistémicos: los primeros se refieren a la oferta educativa, la desigualdad en la calidad de los servicios educativos y los mecanismos de acceso, asociados a la asignación de plantel, modalidad y turno; los factores intrasistémicos se vinculan con las prácticas pedagógicas inadecuadas, formación docente limitada y condiciones laborales precarias, infraestructura y equipamiento insuficiente, incompatibilidad entre la cultura juvenil y escolar, currículo poco pertinente, gestión escolar deficiente, y participación limitada de padres y estudiantes en la escuela.

Otra causa muy común en América Latina tiene que ver con el embarazo en la adolescencia, lo que obliga a los futuros padres a tener que asumir responsabilidades familiares que pueden afectar el desarrollo normal de sus actividades académicas (Mendoza y Subiría, 2013). En este contexto, vale destacar lo señalado por Moreno, Ortiz y González (2016), quienes subrayan las menores oportunidades que tienen las mujeres para estudiar.

Székely (2015), Weiss (2015) y Huerta, Velasco y Jiménez (2016) creen que en la deserción escolar también se debe considerar la escolaridad de los padres e incluso el hecho de que convivan o no con sus hijos, así como ciertos problemas relacionados con la desmotivación, el desinterés por la escuela, las adicciones o la violencia. 


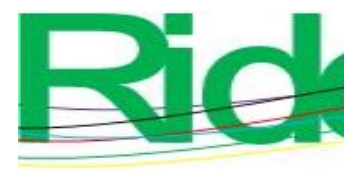

Revista Iberoamericana para la Investigación y el Desarrollo Educativo

ISSN $2007-7467$

Otra de las causas de la deserción escolar es la actitud o la desmotivación del estudiante. Para ello, resulta vital el papel que deben desempeñar tanto las instituciones con sus programas educativos como los docentes con sus prácticas pedagógicas. Es cierto que los problemas de actitud de los estudiantes provienen de su desarrollo como personas, pero también es verdad que es en la trayectoria escolar donde se gesta el desarrollo social. En otras palabras, todas las personas que conforman la institución educativa deben interesarse por los problemas que enfrentan los estudiantes y atenderlos mediante todo tipo de actividades que permitan crear un ambiente de integración (Donoso, Donoso y Arias, 2018).

Aunado a esto, se pueden crear programas de orientación vocacional y motivación hacia la carrera que se estudia, lo cual beneficiaría a los individuos de forma particular y a la sociedad de manera general. El objetivo es mejorar la actitud que tienen las personas para cumplir con sus objetivos, pues de esa manera se puede conseguir el éxito académico y personal (Ortega, 2012). Si se logra un cambio positivo en la mentalidad de los alumnos y se toma en cuenta el desarrollo individual de cada uno, ellos mismos tendrán las herramientas para enfrenar no solo los retos académicos, sino incluso los personales. El reto es prevenir el abandono escolar, pero para ello se deben analizar con detalle los distintos factores que lo pueden generar (Canales y De los Ríos, 2018).

\section{Conclusiones}

El objetivo de este trabajo fue realizar una revisión de literatura para conocer algunas definiciones en torno al concepto deserción escolar, así como sus causas en el nivel superior. Al respecto, se puede decir que esas instituciones deben involucrarse de forma más activa en dicho fenómeno, lo cual se puede lograr actualizando sus programas académicos e incluyendo estrategias didácticas, asesorías y seguimiento a los estudiantes mediante tutorías. Asimismo, se debe trabajar en el aspecto emocional de los estudiantes, para lo cual se debe lograr establecer empatía para facilitar la adaptación, en especial en el primer año universitario. Por último, no se debe olvidar el papel que cumplen los padres en dicho proceso de adaptación, ya que representan un apoyo económico y emocional invaluable para finalizar con éxito el proceso formativo en la universidad.

Es importante analizar los factores que influyen en la deserción escolar a nivel universitario. Para ello, se debe atender a todos los estudiantes que puedan presentar algún tipo de inconveniente, en especial a los de nuevo ingreso, pues estos se enfrentan a un ámbito académico que les exige retos y responsabilidades con los que no están familiarizados. En concordancia con 


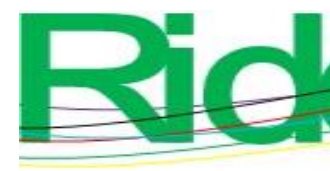

Revista Iberoamericana para la Investigación y el Desarrollo Educativo

ISSN 2007 - 7467

esta idea, Silva-Laya (2011) demuestra que el primer año universitario constituye un tramo crítico en la definición de trayectorias estudiantiles exitosas, pues es en este lapso donde se suele tomar la decisión de abandonar o proseguir con los estudios. Los alumnos, por tanto, deben ser acompañados para que enfrenten las dificultades externas (p. ej., relacionadas con los programas académicos), así como las variables personales vinculadas sobre todo con la autoestima. Para eso, los padres de familia también deben involucrarse, pues ellos representan un apoyo invaluable para los hijos, lo cual impactaría de forma positiva en la motivación, conducta y, por ende, rendimiento académico (Espinosa Hoffmann y Claro Stuardo 2010).

Otra figura que se debe reforzar es la del tutor académico, papel que debe ser desempeñado con el fin de llevar un seguimiento y un análisis del proceso de aprendizaje del alumnado para prevenir la deserción escolar. En tal sentido, los centros educativos deben implementar un plan de acción tutorial que incluya asesoramiento psicológico, lo cual de seguro disminuirá los índices de deserción escolar (Muñoz y Martínez, 2017).

\section{Futuras Líneas de Investigación}

Una vez desarrollada la Investigación, y conociendo a detalle la revisión de literatura sobre la deserción escolar en México, se han descubierto con mayor profundidad líneas de investigación con las que se puede mejorar este tema; a continuación, se presentan algunas:

El tema de Deserción escolar es muy amplio, es primordial que el docente conozca e identifique las causas de deserción de los estudiantes de los diferentes niveles académicos para prevenir a través de su orientación con el alumnado.

Adecuación y actualización de programas curriculares en función de las necesidades de los estudiantes con el fin de lograr la inclusión en el aula y menor rezago escolar.

Formación de la carrera docente, para que los maestros esten mas involucrados en su actividad cotidiana y conozcan mas de cerca la problemática de sus estudiantes, con el fin de evitar la deserción escolar.

Para futuros estudios es importante concretar programas de evaluación del desempeño de los docentes y de los alumnos para identificar posibles fugas de talento y evitar la deserción escolar en México. 


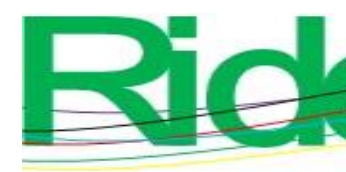

Revista Iberoamericana para la Investigación y el Desarrollo Educativo

ISSN $2007-7467$

\section{Referencias}

Arango, L. G. (2007). Cuestión de supervivencia. Graduación, deserción y rezago en la Universidad Nacional de Colombia. Teoría y Praxis Investigativa, 2(2), 74-77.

Canales, A. y De los Ríos, D. (2007). Factores explicativos de la deserción universitaria. Calidad en la Educación, (26), 173-201.

De Witte, K. and Rogge, N. (2013). Dropout from secondary education: All's well that begins well. European Journal of Education, 48(1), 131-149.

Del Castillo, M. H. (2012). Causas, consecuencias y prevención de la deserción escolar: un manual de auto ayuda para padres, maestros y tutores. Palibrio.

Departamento Nacional de Estadística [DANE] (2016). Departamento Administrativo Nacional de Estadísticas. Recuperado de http://www.dane.gov.co/index.php/estadisticas-portema/educacion

Donoso, S., Donoso, G. y Arias, Ó. (2018). Iniciativas de retención de estudiantes de educación superior. Calidad en la Educación, (33), 15-61.

Donoso, S. y Schiefelbein, E. (2012). Análisis de los modelos explicativos de retención de estudiantes en la universidad: una visión desde la desigualdad social. Estudios Pedagógicos, $33(1), 7-27$.

Espinosa Hoffmann, V. y Claro Stuardo, J. P. (2010). Estrategias de prevención de la deserción en la educación secundaria: perspectiva latinoamericana. Revista de Educación, (1), 257-280.

Fernández, T. (2010). La desafiliación en la educación media y superior de Uruguay: conceptos, estudios y políticas. Comisión Sectorial de Investigación Científica. Universidad de la República.

Franklin, B. and Kochan, S. (2000). Collecting and reporting dropout data in Lousiana. Estudio presentado en la conferencia anual del American Education Research Association. Los Angeles.

Gómez, A. (1998). Educación: la agenda del siglo XXI: hacia un desarrollo humano. Colombia: PNUD. TM Editores.

Huerta, P., Velasco, M. y Jiménez, M. (2016). Causas de la deserción escolar en las telesecundarias de la zona 55. Revista Huella de la Palabra, (8).

Instituto Nacional para la Evaluación de la Educación [INEE] (2017). Directrices para mejorar la permanencia escolar en la educación media superior. Instituto Nacional para la Educación 




\section{Revista Iberoamericana para la Investigación y el Desarrollo Educativo ISSN 2007 - 7467}

Nacional en México. Recuperado de https://www.inee.edu.mx/directrices-paramejorar/directrices-para-mejorar-la-permanencia escolar-en-la-educacion-media-superior/ Latiesa, (2011). Tipología y causas de la deserción universitaria y el retraso en los estudios. España: Universidad de Granada.

Lavado, P. y Gallegos, J. (2005). La dinámica de la deserción escolar en el Perú: un enfoque usando modelos de duración. Lima, Perú: Centro de Investigación de la Universidad del Pacífico.

Mendoza, W. y Subiría, G. (2013). El embarazo adolescente en el Perú: situación actual e implicancias para las políticas públicas. Revista Peruana de Medicina Experimental y Salud Pública, 30(3), 471-479.

Moreno Bernal, D. M. (2013). La deserción escolar: un problema de carácter social. Revista in Vestigium Ire, 6(1), 115-124.

Moreno Torres, M. A., Ortiz, Y. y González, M. (2016). Capacitación de docentes en procesos neurocognitivos para atender la deserción escolar asociada a aprovechamiento académico. Revista Puertorriqueña de Psicología, 27(2), 304-318.

Muñoz, J. S. y Martínez, A. A. (2017). El problema de la deserción escolar en la producción científica educativa. Revista Internacional de Ciencias Sociales y Humanidades, SOCIOTAM, 27(1), 89-112.

Ortega, F. (2012). Los desertores del futuro (vol. 5). Centro de Estudios Avanzados: Universidad Nacional de Córdoba.

Ortiz-Hernández, E. (2018). Retos y perspectivas del currículo integrado. Cuaderno de Investigación en la Educación, (21), 35-56.

Páramo, G. J. y Correa, C. A. (2012). Deserción estudiantil universitaria. Conceptualización. Revista Universidad EAFIT, 35(114), 65-78.

Rinne, R. and Järvinen, T. (2011). Dropout and Completion in Upper Secondary Education in Finland. School Dropout and Completion: International comparative estudies in theory and policy, 1(2), 215-232. Doi: 10.1007/978-90-481-9763-7_12

Román, M. C. (2013). Factores asociados al abandono y la deserción escolar en América Latina: una mirada en conjunto. Revista Iberoamericana sobre Calidad, Eficacia y Cambio en Educación, 11(2), 33-59.

Rosado-Ortiz, G. (2012). Deserción o exclusión escolar: análisis sobre educación, desigualdad y pobreza en Puerto Rico. Revista Paideia Puertorriqueña, 7(2). 


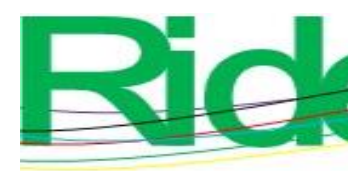

Revista Iberoamericana para la Investigación y el Desarrollo Educativo

ISSN $2007-7467$

Santamaría, F. and Bustos, A. (2013). Permanence and Dropout Rates in Higher Education: A Research Experience Based on Young Students Voices. Revista Infancias Imágenes, 12(2) 73-80.

Silva-Laya, M. (2011). El primer año universitario: un tramo crítico para el éxito académico. Perfiles Educativos, 33, 102-114.

Spady, W. G. (1970). Dropouts from higher education: An interdisciplinary review and synthesis. Interchange, 1(1), 64-85. Doi: https://doi.org/10.1007/BF02214313

Székely, M. (2015). Estudio sobre los principales resultados y recomendaciones de la investigación y evaluación educativa en el eje de prevención y atención a la deserción escolar en educación media superior. México: INEE.

Torres, L. E. (2012). Retención estudiantil en la educación superior: revisión de la literatura y elementos de un modelo para el contexto colombiano. Bogotá, D. C.: Editorial Pontificia Universidad Javeriana.

Vázquez, N. V. (2012). Factores que inciden en la deserción escolar: hacia un modelo descriptivo para líderes educativos (disertación doctoral). Available from ProQuest Dissertations and Theses (UMI No. 3509714).

Vásquez Velásquez, J., Castaño Vélez, E., Gallón Gómez, S. A. y Gómez Portilla, K (2003). Determinantes de la deserción estudiantil en la Universidad de Antioquia. Borradores del CIE, (04), 1-38. Recuperado de http://hdl.handle.net/10495/3627

Vries, W. D., León Arenas, P., Romero Muñoz, J. F. y Hernández Saldaña, I. (2011). ¿Desertores o decepcionados? Distintas causas para abandonar los estudios universitarios. Revista de la Educación Superior, 40(160), 29-49.

Weiss, E. (2015). El abandono escolar en la educación media superior: dimensiones, causas y políticas para abatirlo. En Ramírez R. (ed.), Desafíos de la educación media superior (pp. 81-160). Ciudad de México: Instituto Belisario Domínguez. 